\title{
Scattering of nitrogen molecules by silver atoms
}

\section{Citation}

Loreau, Jérôme, Peng Zhang, and Alexander Dalgarno. 2012. "Scattering of Nitrogen

Molecules by Silver Atoms." The Journal of Chemical Physics 136 (16) (April 28): 164305. doi:10.1063/1.3703518.

\section{Published Version}

doi:dx.doi.org/10.1063/1.3703518

\section{Permanent link}

http://nrs.harvard.edu/urn-3:HUL.InstRepos:30403727

\section{Terms of Use}

This article was downloaded from Harvard University's DASH repository, and is made available under the terms and conditions applicable to Other Posted Material, as set forth at http:// nrs.harvard.edu/urn-3:HUL.InstRepos:dash.current.terms-of-use\#LAA

\section{Share Your Story}

The Harvard community has made this article openly available.

Please share how this access benefits you. Submit a story.

Accessibility 


\title{
Scattering of nitrogen molecules by silver atoms
}

\author{
Jérôme Loreau, ${ }^{1, a)}$ Peng Zhang, ${ }^{1,2}$ and Alexander Dalgarno ${ }^{1}$ \\ ${ }^{1}$ Institute for Theoretical Atomic, Molecular and Optical Physics (ITAMP), Harvard-Smithsonian \\ Center for Astrophysics, Cambridge, Massachusetts 02138, USA \\ ${ }^{2}$ Department of Chemistry, Duke University, Durham, North Carolina 27708, USA
}

(Received 24 February 2012; accepted 29 March 2012; published online 25 April 2012)

\begin{abstract}
We present a quantal study of the rotationally elastic and inelastic scattering of $\mathrm{Ag}$ and $\mathrm{N}_{2}$, with the nitrogen molecule treated as a rigid rotor. The two-dimensional potential energy surface of the $\mathrm{AgN}_{2}$ complex is obtained ab initio by means of the spin unrestricted coupled-cluster method with single, double, and perturbative triple excitations. The global minimum is found to be located at an internuclear distance of $8.13 a_{0}$ and an angle of $127.2^{\circ}$. The long-range part of the potential is constructed from the dynamic electric dipole polarizabilities of $\mathrm{Ag}$ and $\mathrm{N}_{2}$. Elastic, excitation, and relaxation cross sections and rates are calculated for energies between 0.1 and $5000 \mathrm{~cm}^{-1}$. The momentum transfer cross sections and rates are also computed. Finally, we compare the cross sections for $\mathrm{Ag}-$ $\mathrm{N}_{2}$ and $\mathrm{Na}-\mathrm{N}_{2}$ to explore the possibility of using silver instead of sodium in experimental tests. (C) 2012 American Institute of Physics. [http://dx.doi.org/10.1063/1.3703518]
\end{abstract}

\section{INTRODUCTION}

Sodium laser guide stars are currently in operation or under development at major ground-based telescopes in order to improve the use of adaptive optics. One of the major difficulties in their implementation is the modeling of the photon return from the sodium layer present in the mesosphere. In particular, collisions of $\mathrm{Na}$ with $\mathrm{N}_{2}, \mathrm{O}$, and $\mathrm{O}_{2}$ and spin exchange collisions with $\mathrm{O}_{2}$ change the velocity of the sodium atoms, hence, these processes must be included in theoretical models in order to optimize the return flux. ${ }^{1}$ In addition, it is also important to consider the effects of these collisions in the context of the use of mesospheric sodium in remote-detection magnetometry. ${ }^{2}$ We have recently performed a theoretical investigation of $\mathrm{Na}-\mathrm{N}_{2}$ collisions using the close-coupling method for temperatures between 100 and $300 \mathrm{~K},{ }^{3}$ but the cross sections for collisions of $\mathrm{Na}$ with $\mathrm{O}$ and $\mathrm{O}_{2}$ are still unknown. Furthermore, the experimental determination of these cross sections is very challenging due to the high reactivity of sodium. Due to its low chemical reactivity, and because their electronic structure are similar (in the ground state, a single $s$ electron outside a filled shell), silver has been proposed as a possible substitute for sodium in experiments. ${ }^{4}$

Silver atoms ablated into a dense buffer gas such as $\mathrm{He}$ or $\mathrm{N}_{2}$ at temperatures ranging from a few $\mathrm{K}$ to room temperature have been explored experimentally and proposed as a potential system for a high-sensitivity cryogenic atomic magnetometer. ${ }^{5} \mathrm{Ag}-\mathrm{N}_{2}$ collisions could also have applications in the context of spin-exchange optical pumping (SEOP). It has been recently demonstrated theoretically that the rate of spin polarization of ${ }^{3} \mathrm{He}$ can be improved by the use of $\mathrm{Ag}$ instead of alkali-metal atoms. ${ }^{6}$ A typical SEOP experiment consists of a cell containing an alkali-metal and a noble gas. The alkali-metal is spin polarized by optical pumping, and the spin polarization is transferred to the noble gas

\footnotetext{
a)Electronic mail: jloreau@cfa.harvard.edu.
}

during collisions. ${ }^{7}$ The cell also contains $\mathrm{N}_{2}$, which is used to quench the excited atoms, and the interaction of nitrogen with the alkali-metal can have important effects on the SEOP mechanism. ${ }^{8}$

The purpose of this work is to describe $\mathrm{Ag}-\mathrm{N}_{2}$ collisions using a fully quantal method. In Sec. II, we present the twodimensional potential energy surface of the ground state of the $\mathrm{AgN}_{2}$ van der Waals complex, calculated using ab initio methods with the $\mathrm{N}_{2}$ molecule frozen in its equilibrium geometry. In Sec. III, we calculate the elastic and inelastic (excitation and de-excitation) rotational cross sections, as well as the differential and momentum transfer cross sections, for energies in the range $0.1-5000 \mathrm{~cm}^{-1}$. We also calculate the corresponding rate constants for temperatures between $1 \mathrm{~K}$ and $1000 \mathrm{~K}$, and we compare these results to the calculations recently reported on $\mathrm{Na}-\mathrm{N}_{2}$ scattering ${ }^{3}$ to investigate the possibility of using $\mathrm{Ag}$ as a substitute for $\mathrm{Na}$ in experimental tests.

\section{AB INITIO CALCULATIONS}

\section{A. Interaction potential}

To parametrize the potential energy surface of the $\mathrm{AgN}_{2}$ complex, we used the three standard Jacobi coordinates $(R$, $r, \theta) . R$ is the distance between the silver atom and the center of mass of the $\mathrm{N}_{2}$ molecule, $r$ denotes the length of the $\mathrm{N}-\mathrm{N}$ bond, and $\theta$ is the angle between $R$ and $r$. As the first excited vibrational state of $\mathrm{N}_{2}$ lies about $2330 \mathrm{~cm}^{-1}$ above the ground state, ${ }^{9}$ vibrational excitation is not expected to contribute significantly. The internuclear distance of $\mathrm{N}_{2}$ is therefore fixed to the equilibrium geometry, $r=2.4132$ a.u. The ground state configuration of the silver atom is $4 d^{10} 5 s^{12} S$, while the ground state of the nitrogen molecule is a ${ }^{1} \Sigma_{\mathrm{g}}^{+}$state. Therefore, the ground state of the $\mathrm{AgN}_{2}$ van der Waals complex corresponds to the ${ }^{2} A^{\prime}$ representation of the symmetry group $C_{s}$. For the particular geometries $\theta=0^{\circ}$ (linear) and 
$\theta=90^{\circ}$ (T-shaped), the ground state will be, respectively, a ${ }^{2} \Sigma^{+}$or a ${ }^{2} A_{1}$ state.

The two-dimensional potential energy surface (PES) of the ground state was calculated using the spin unrestricted coupled-cluster method with single, double, and perturbative triple excitations $(\mathrm{UCCSD}(\mathrm{T}))^{10,11}$ implemented in the MOLPRO 2009.1 package. $^{12}$ The reference wave function employed in the CC calculations was generated from the spin restricted Hartree-Fock method. For the Ag atom, we used the cc-pwCVQZ-PP basis set. ${ }^{13}$ This basis set consists of a small-core relativistic pseudopotential, which replaces the $1 s$ $-3 d$ core and includes scalar and spin-orbit effects, ${ }^{14}$ and of a quadruple zeta basis set for the valence electrons, including valence plus core-valence correlation. This basis set was augmented by diffuse functions ${ }^{13}$ (one for each angular momentum symmetry) to accurately describe the weakly bound complex $\mathrm{AgN}_{2}$. For the $\mathrm{N}$ atoms, the aug-cc-pwCVQZ basis set $^{15}$ was used. In our calculations, we treated the $1 s^{2}$ electrons of both $\mathrm{N}$ atoms as core electrons. In total, 29 electrons (the $4 s^{2} 4 p^{6} 4 d^{10} 5 s^{1}$ electrons of the $\mathrm{Ag}$ atom and the $2 s^{2} 2 p^{3}$ electrons of the $\mathrm{N}$ atoms) were correlated explicitly in the $\mathrm{UCCSD}(\mathrm{T})$ calculations.

We constructed the two-dimensional PES $V(R, \theta)$ using an uniform grid of 583 geometries. The grid for the intermolecular distance is $3.4 \leq R \leq 19$ a.u. with a step-length of 0.3 a.u. while for the $\theta$ we used a grid $90^{\circ} \leq \theta \leq 180^{\circ}$ with a step size of $10^{\circ}$, with an additional series of points at $\theta=95^{\circ}$. The potential for $0^{\circ} \leq \theta<90^{\circ}$ is obtained by symmetry with respect to $\theta=\pi / 2$. For each geometry, the energy was corrected to account for the basis set superposition error (BSSE) using the counterpoise method. ${ }^{16}$

The $a b$ initio potential energy surface $V(R, \theta)$ is shown in Fig. 1. It is strongly repulsive at distances smaller than 6 a.u., and weakly attractive for large $R$. The potential has a global minimum at $\theta=127.2^{\circ}$ (and at $\theta=52.8^{\circ}$ ) and the equilibrium distance is $R=8.13$ a.u., with a dissociation energy $D_{e}=81.8 \mathrm{~cm}^{-1}$. As can be seen from Fig. 1 , the potential has a saddle point at $\theta=90^{\circ}$. The dissociation energy of this configuration is $73.1 \mathrm{~cm}^{-1}, 8.7 \mathrm{~cm}^{-1}$ above the global minimum, and corresponds to the intermolecular distance $R$ $=8.07$ a.u. In the linear geometry $\left(\theta=0^{\circ}\right.$ or $\left.180^{\circ}\right)$, the minimum of the potential is shifted toward larger intermolecular distances $(R=8.83$ a.u.) and the dissociation energy is $67.0 \mathrm{~cm}^{-1}$. The distances $R$ for which the interaction energy is maximal are given in Table I for a series of angles. In particular, we observe that the equilibrium distance increases as the complex approaches the linear configuration. The effect of the BSSE on the dissociation energy at the global minimum is about $12 \mathrm{~cm}^{-1}$.

\section{B. Long-range interactions}

The asymptotic long-range attractive potential $V_{\text {as }}$ is dominated by the dispersion forces and can be constructed in an analytical form. We retain here the leading term,

$$
V_{\mathrm{as}}=-\frac{C_{6}}{R^{6}}
$$

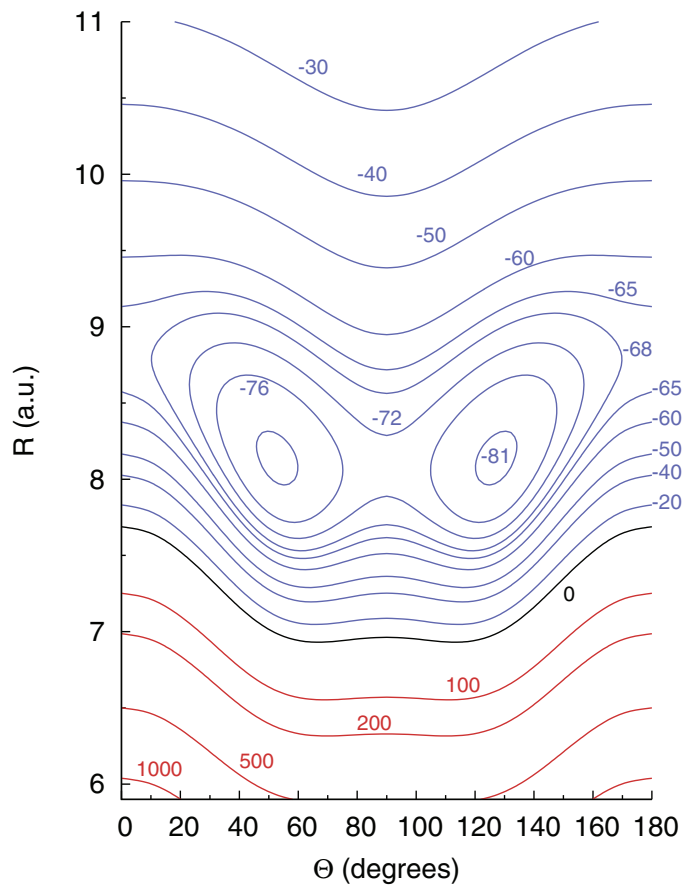

FIG. 1. Contour plot of the potential energy surface $V(R, \theta)$ for the $\mathrm{AgN}_{2}$ complex. The energies are in units of $\mathrm{cm}^{-1}$.

In the case of the interaction between an $S$-state atom and a linear molecule, the $C_{6}$ coefficient is given by the sum of an isotropic and an anisotropic component, ${ }^{17}$

$$
C_{6}=C_{6,0} P_{0}(\cos \theta)+C_{6,2} P_{2}(\cos \theta) \text {, }
$$

where $P_{0}$ and $P_{2}$ are the Legendre polynomials of order 0 and 2 , respectively. The isotropic and anisotropic dispersion coefficients may be derived using the dynamic electric dipole polarizabilities as

$$
\begin{gathered}
C_{6,0}=\frac{3}{\pi} \int_{0}^{\infty} \alpha^{\mathrm{Ag}}(i \omega) \bar{\alpha}^{\mathrm{N}_{2}}(i \omega) d \omega \\
C_{6,2}=\frac{1}{\pi} \int_{0}^{\infty} \alpha^{\mathrm{Ag}}(i \omega)\left[\alpha_{\|}^{\mathrm{N}_{2}}(i \omega)-\alpha_{\perp}^{\mathrm{N}_{2}}(i \omega)\right] d \omega
\end{gathered}
$$

TABLE I. Equilibrium distances of the $\mathrm{Ag}-\mathrm{N}_{2}$ potential $V(R, \theta)$ for various values of $\theta$ and with $r=2.4132$ a.u., and corresponding dissociation energies $D_{e} . \theta=127.2^{\circ}$ is the global minimum of the potential.

\begin{tabular}{lcc}
\hline \hline$\theta(\mathrm{deg})$ & $R$ (a.u.) & $D_{e}\left(\mathrm{~cm}^{-1}\right)$ \\
\hline 90 & 8.07 & 73.1 \\
100 & 8.06 & 74.5 \\
110 & 8.06 & 77.8 \\
120 & 8.07 & 81.0 \\
127.2 & 8.13 & 81.8 \\
130 & 8.16 & 81.7 \\
140 & 8.31 & 79.2 \\
150 & 8.50 & 75.0 \\
160 & 8.67 & 70.8 \\
170 & 8.79 & 68.0 \\
180 & 8.83 & 67.0 \\
\hline \hline
\end{tabular}


TABLE II. Parameters of the switching function (5).

\begin{tabular}{lccc}
\hline \hline$a$ & $b$ & $c$ & $d$ \\
\hline-0.0216922 & 0.717031 & 0.124343 & 19.6663 \\
\hline
\end{tabular}

where $\alpha_{\|}(i \omega)$ and $\alpha_{\perp}(i \omega)$ are, respectively, the longitudinal and transverse dynamical electric dipole polarizabilities, evaluated as functions of imaginary frequencies $i \omega$, while $\bar{\alpha}$ is the average dipole polarizability, $\bar{\alpha}=\frac{1}{3}\left(\alpha_{\|}+2 \alpha_{\perp}\right)$. The expressions (3) and (4) can be calculated efficiently using Gaussian quadrature. ${ }^{18}$ As in Ref. 3, the dynamic polarizabilities of the $\mathrm{N}_{2}$ molecule were evaluated using the time-independent linear response coupled-cluster theory ${ }^{19}$ at the third-order in the fluctuation potential, as implemented in MOLPRO, and a triply augmented correlation consistent polarized core-valence sextuple zeta (t-aug-cc-pCV6Z) basis set. ${ }^{20}$

For the Ag atom, we adopted the accurate values reported in Ref. 21, which include core contributions. The integration was realized using a 50-point Gaussian quadrature, and we obtained $C_{6,0}=147.56$ a.u. and $C_{6,2}=17.47$ a.u. The accuracy of these dispersion coefficients can be further verified through direct comparison with the $a b$ initio $\operatorname{UCCSD}(\mathrm{T})$ calculations. Substituting the values of the $C_{6}$ coefficients into Eq. (1), we find that the energy difference between the UCCSD(T) calculations and the asymptotic potential (1) at the last point of our computed grid ( $R=19$ a.u.) is smaller than $0.2 \mathrm{~cm}^{-1}$, the largest difference occurring for the linear geometry $\left(\theta=180^{\circ}\right)$. In order to smoothly connect the ab initio points and the asymptotic potential, we used a switching function expressed as the product of an $R$-dependent and a $\theta$-dependent function. It is given by

$$
f(R, \theta)=-(1+\tanh (a R+b)) \cos (c(\theta+d)) .
$$

The four parameters $a, b, c, d$ were optimized with respect to the $a b$ initio points at large $R$ using a nonlinear least-squares Marquardt-Levenberg algorithm and are given in Table II. The root mean square deviation was $3 \times 10^{-3} \mathrm{~cm}^{-1}$. A cubic spline method was employed to generate the potential for $R$ $<19$ a.u.

In scattering calculations, it is often useful to expand the intermolecular potential $V(R, \theta)$ in terms of Legendre polynomials of order $\lambda$ in order to simplify the evaluation of its matrix elements,

$$
V(R, \theta)=\sum_{\lambda} V_{\lambda}(R) P_{\lambda}(\cos \theta),
$$

where the $V_{\lambda}(R)$ are known as "radial strength functions". The first four radial functions $V_{\lambda}(R)$ are shown in Fig. 2. Only even values of $\lambda$ appear in (6) due to the symmetry of the intermolecular potential.

\section{SCATTERING CALCULATIONS}

\section{A. Computational method}

We calculated the cross sections for $\mathrm{Ag}-\mathrm{N}_{2}$ collisions using the quantum close-coupling method developed by Arthurs and Dalgarno, ${ }^{22}$ which has been described extensively in the

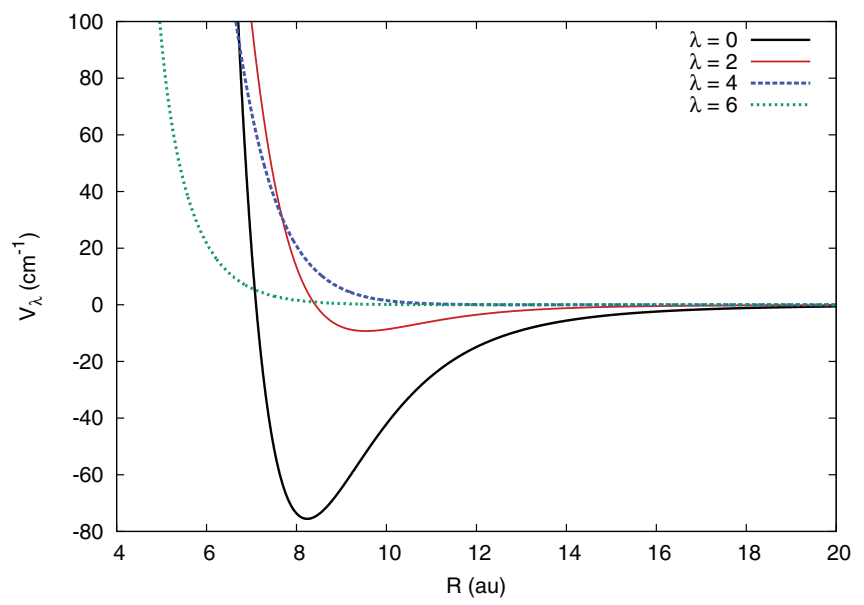

FIG. 2. The first four radial functions $V_{\lambda}(R)$ in the expansion (6) of the intermolecular potential $V(R, \theta)$.

literature. ${ }^{23} \mathrm{~N}_{2}$ was held fixed at its equilibrium bond-length and treated in the rigid-rotor approximation. We represented the energy of the rotational levels of the nitrogen ${ }^{1} \Sigma_{\mathrm{g}}^{+}$ground state using the rotational constants of $\mathrm{N}_{2}$ given in Refs. 9 and 24 ,

$$
\begin{aligned}
& B_{e}=1.99824 \mathrm{~cm}^{-1}, \quad D_{e}=5.76 \cdot 10^{-6} \mathrm{~cm}^{-1} \\
& \alpha_{e}=0.017318 \mathrm{~cm}^{-1} .
\end{aligned}
$$

In the close-coupling method, the total wave function of the $\mathrm{Ag}-\mathrm{N}_{2}$ complex is expanded as a sum of products of radial and rotational functions. When inserted in the Schrödinger equation, this leads to a set of second-order coupled differential equations for the radial functions that must be solved with appropriate boundary conditions. In these equations, the coupling between the different channels is represented by matrix elements of the intermolecular potential. It is convenient to introduce the total angular momentum $\mathbf{J}=\mathbf{j}+\mathbf{l}$, where $\mathbf{j}$ is the angular momentum of $\mathrm{N}_{2}$ and $\mathbf{l}$ is the orbital momentum. The total angular momentum is conserved during the collision, so that the coupled equations are block-diagonal in $J$. The $S$ matrix elements $S_{j^{\prime} l^{\prime} j l}^{J}$ can then be obtained from the asymptotic behavior of the radial functions, and the cross section for the transition from an initial rotational state $j$ to a final rotational state $j^{\prime}$ is given in terms of the $S$ matrix elements as

$$
\begin{aligned}
\sigma_{j \rightarrow j^{\prime}}= & \frac{\pi}{(2 j+1) k_{j}^{2}} \sum_{J=0}^{\infty} \sum_{l=|J-j|}^{|J+j|} \sum_{l^{\prime}=\left|J-j^{\prime}\right|}^{\left|J+j^{\prime}\right|} \\
& \times(2 J+1)\left|\delta_{j j^{\prime}} \delta_{l l^{\prime}}-S_{j^{\prime} l^{\prime} j l}^{J}\right|^{2},
\end{aligned}
$$

where $k_{j}^{2}=2 \mu E_{c}=2 \mu\left(E-\epsilon_{j}\right)$ is the wavenumber in the entrance channel with energy $\epsilon_{j}, E_{c}$ is the kinetic energy, and $E$ is the total (kinetic plus rotor) energy.

In addition to the integral cross section, the properties of the atom-molecule collision are also determined by the momentum transfer cross section $\sigma_{j \rightarrow j^{\prime}}^{\text {tr }}$ (or transport cross section). The momentum transfer cross section from an initial level $j$ to a final level $j^{\prime}$ is obtained from the differential cross 
sections $d \sigma / d \Omega$ as $^{25}$

$$
\sigma_{j \rightarrow j^{\prime}}^{\mathrm{tr}}\left(E_{c}\right)=2 \pi \int \frac{d \sigma_{j \rightarrow j^{\prime}}\left(E_{c}, \gamma\right)}{d \Omega} \sin \gamma \Phi\left(E_{c}, \gamma\right) d \gamma,
$$

where $\gamma$ is the scattering angle. It differs from the integral cross section given by Eq. (7) by the function

$$
\Phi\left(E_{c}, \gamma\right)=1-\sqrt{E_{c}^{\prime} / E_{c}} \cos \gamma
$$

where $E_{c}^{\prime}$ is the final kinetic energy, related to the initial kinetic energy by

$$
E_{c}^{\prime}=E_{c}+\epsilon_{j}-\epsilon_{j^{\prime}}
$$

The thermal rate coefficient for a transition $j \rightarrow j^{\prime}$ at a given temperature $T$ is given by the integral of the cross section over a Maxwell-Boltzmann distribution of initial energies

$$
k_{j \rightarrow j^{\prime}}(T)=\left(\frac{2}{k_{B} T}\right)^{3 / 2} \frac{1}{\sqrt{\pi \mu}} \int_{0}^{\infty} E_{c} e^{-E_{c} / k_{B} T} \sigma_{j \rightarrow j^{\prime}}\left(E_{c}\right) d E_{c},
$$

where $k_{B}$ is the Boltzmann constant. The rate coefficient for momentum transfer, $k^{\operatorname{tr}}(T)$, is given by the same expression, replacing $\sigma\left(E_{c}\right)$ by $\sigma^{\operatorname{tr}}\left(E_{c}\right)$.

We carried out the scattering calculations for collision energies between 0.1 and $5000 \mathrm{~cm}^{-1}$ using the nonreactive scattering code MOLSCAT of Hutson and Green. ${ }^{26}$ The radial equations were solved using a hybrid modified log-derivative Airy propagator. ${ }^{27}$ The log-derivative matrix is propagated on a grid of intermolecular distances between $R_{\min }$ and $R_{\text {max }}$, where the numerical calculations are matched with the asymptotic solutions and the $S$ matrix elements are extracted. We used a grid starting at $R_{\min }=3$ a.u., while the $R_{\max }$ was in the range 70-150 a.u., depending on the energy of collision. For a particular energy, the propagation is carried out until convergence of the sum over the total angular quantum number $J$ in Eq. (7) is achieved.

\section{B. Cross sections}

In this section, we present the integral and momentum transfer cross sections for elastic and rotationally inelastic transitions. The elastic and inelastic integral cross sections starting from the initial rotational levels $j=1, j=5$, and $j$ $=10$ are shown in Figs. 3-5 for energies between 0.1 and $5000 \mathrm{~cm}^{-1}$. We observe resonance structures in the elastic cross section for the initial rotational level $j=1$, as well as in the inelastic cross sections from $j=1$ to $j^{\prime}=3$ and $j^{\prime}=5$, for energies below the depth of the van der Waals potential $\left(\sim 80 \mathrm{~cm}^{-1}\right)$. These resonances at low kinetic energy are a consequence of the attractive potential well, which allows Ag to be temporarily trapped and hence quasi-bound states to be formed. These states may arise from the tunneling from the centrifugal barrier and from excitation of $\mathrm{N}_{2}$ to an asymptotically closed channel where $\mathrm{N}_{2}$ becomes temporarily trapped in one of the bound states of the potential well. ${ }^{28-30}$ Compared to the depth of the potential well, the energy spacing of the $\mathrm{N}_{2}$ rotational structure is small, so that both types of quasi-bound states occur in the same energy range. Although the resonances are still present for the initial rotational levels

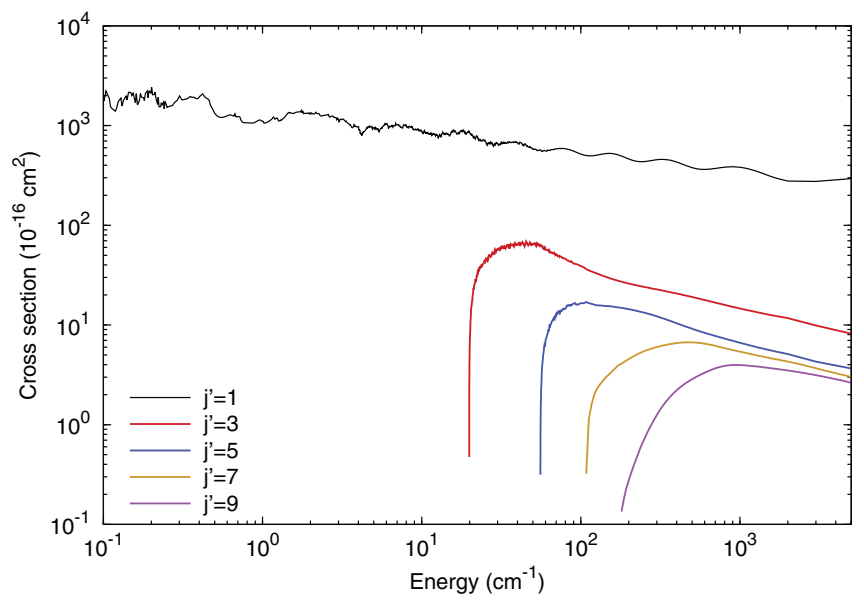

FIG. 3. Elastic and inelastic cross sections $\sigma_{j \rightarrow j^{\prime}}$ for the initial rotational level $j=1$ and the final rotational levels $j^{\prime}=1-9$.

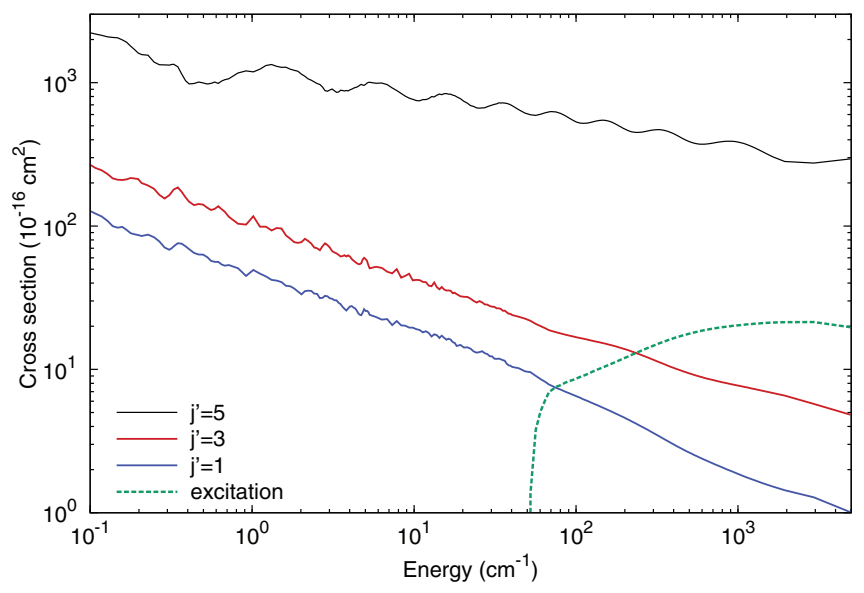

FIG. 4. Elastic and inelastic cross sections $\sigma_{j \rightarrow j^{\prime}}$ for the initial rotational level $j=5$. The excitation cross section is the sum of the cross sections over all rotational levels $j^{\prime}>j$.

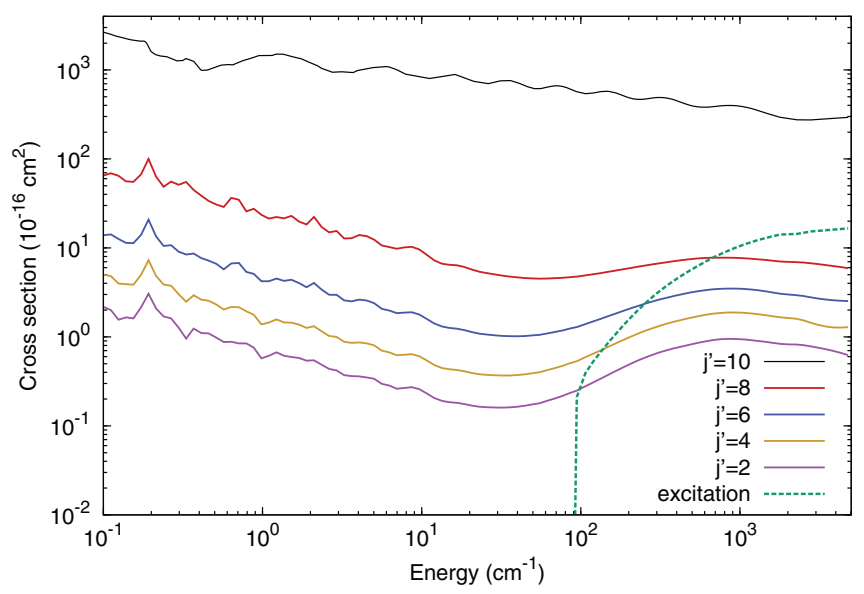

FIG. 5. Elastic and inelastic cross sections $\sigma_{j \rightarrow j^{\prime}}$ for the initial rotational level $j=10$. The excitation cross section is the sum of the cross sections over all rotational levels $j^{\prime}>j$. 


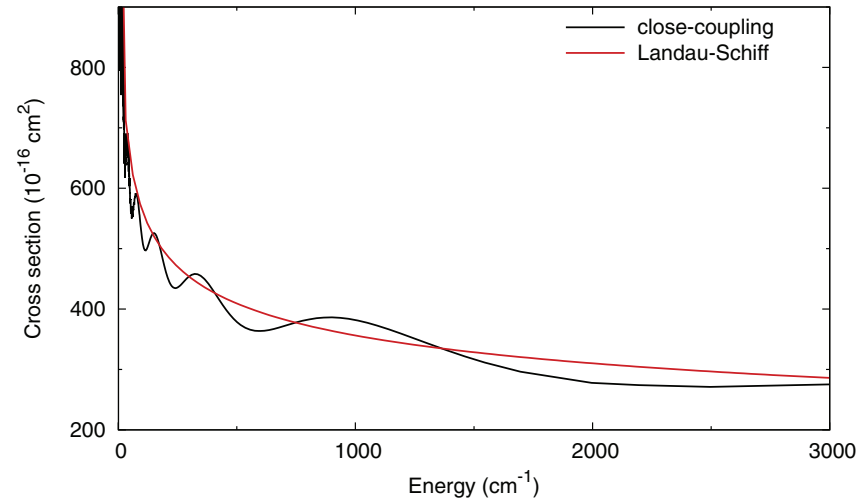

FIG. 6. Elastic cross sections for $j=1$ and comparison with the LandauSchiff formula, Eq. (12).

$j=5$ and $j=10$ (see Figs. 4 and 5), they are gradually suppressed with increase in the initial rotational level, as the van der Waals potential well supports only a limited number of excited quasi-bound levels. Levels corresponding to higher $j$ therefore become virtual states producing no resonances.

In addition to the resonance structure for energies below $80 \mathrm{~cm}^{-1}$, the elastic cross sections present undulations as a function of the kinetic energy. These undulations are present for all initial rotational levels, and the position of the maxima is almost independent of $j$. This peak structure is characteristic of glory undulations, ${ }^{31}$ and the independence of the peak positions with respect to the initial rotational state indicates that the rotational time of $\mathrm{N}_{2}$ is short compared to the characteristic translational time. The elastic cross sections are very similar for all initial rotational levels, although for energies below $80 \mathrm{~cm}^{-1}$, there can be significant differences due to the resonance structure. They become comparable at higher energies, but for a given energy the cross section increases slightly with $j$. At $100 \mathrm{~cm}^{-1}$, the $j=5$ and $j=15$ cross sections are larger than the $j=1$ cross section by about $3 \%$ and $6 \%$, respectively, while the difference drops to $1 \%$ and $5 \%$ at $1000 \mathrm{~cm}^{-1}$.

The elastic cross section can be estimated from the semi-classical Landau-Schiff formula for scattering by a $R^{-n}$ potential, ${ }^{32}$

$\sigma=2 \pi^{\frac{n}{n-1}} \sin \left[\frac{\pi}{2} \frac{n-3}{n-1}\right] \Gamma\left[\frac{n-3}{n-1}\right]\left(\frac{\Gamma\left[\frac{n-1}{2}\right]}{\Gamma\left[\frac{n}{2}\right]}\right)^{\frac{2}{n-1}}\left(\frac{C_{n}}{v}\right)^{\frac{2}{n-1}}$.

The Landau-Schiff elastic cross section, computed from the $C_{6}$ coefficient presented in Sec. II B, is shown in Fig. 6. We observe a very good agreement with the close-coupling calculations for the rotational state $j=1$ illustrated in the figure. As the elastic cross sections are similar for all initial rotational levels, the agreement is valid for all values of $j$.

Over the range of energies considered in this work, the collision is mostly elastic. As can be seen from Figs. 3-5, the inelastic cross sections are roughly an order of magnitude smaller than the elastic. At low energy, the inelastic process is dominated by the relaxation to the rotational level $j^{\prime}=j-2$. At higher energy, when several excited rotational levels become energetically available, the inelastic transitions are dominated by excitation into the rotational levels $j^{\prime}>j$. The rotational relaxation occurs preferentially through

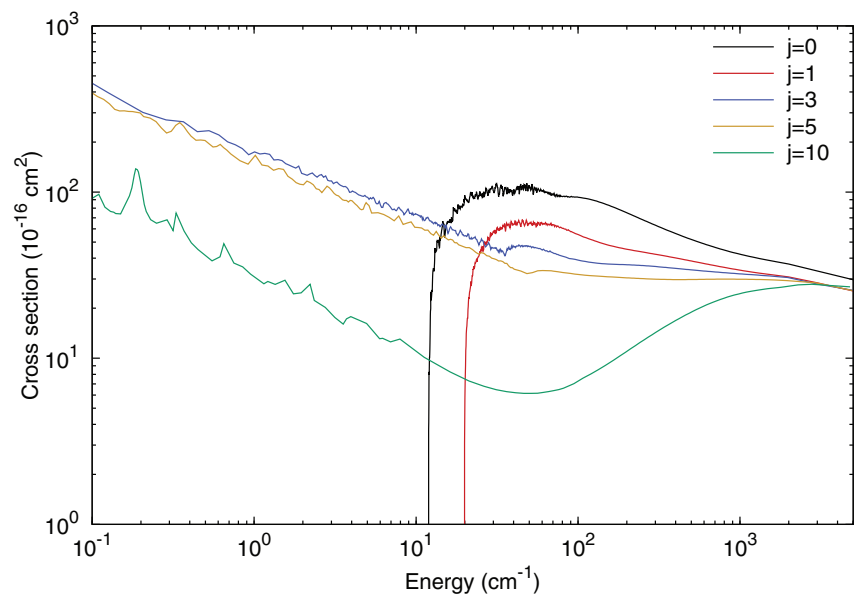

FIG. 7. Total rotationally inelastic cross section $\sum_{j^{\prime} \neq j} \sigma_{j \rightarrow j^{\prime}}$ for several initial rotational levels $j$.

the highest rotational level for all energies. In Fig. 7, we compare the total inelastic (relaxation plus excitation) cross sections for various initial rotational levels. We observe that for a given energy, the inelastic cross sections decrease with increasing initial rotational level. However, at high energies (above $1000 \mathrm{~cm}^{-1}$ ), the cross section includes contributions from many rotational levels and the inelastic cross section becomes independent of the initial rotational state.

The elastic and inelastic momentum transfer cross sections $\sigma_{j \rightarrow j^{\prime}}^{\text {tr }}$, given by Eq. (8) are presented in Fig. 8 for the initial levels $j=1, j=5$, and $j=10$. For energies below the depth of the potential, the elastic cross sections for the various initial rotational levels can be quite different. However, at higher energies they all converge towards the same value. The elastic momentum transfer cross sections are smaller than the elastic integral cross sections at all energies, due to the function $\Phi\left(E_{c}, \gamma\right)=1-\sqrt{E_{c}^{\prime} / E_{c}} \cos \gamma$ (see Eq. (9)), which suppresses the contributions from the small angles (forward scattering) in the differential cross section. The difference between the two types of elastic cross sections increases with the energy. This is due to the fact that at low energy, the

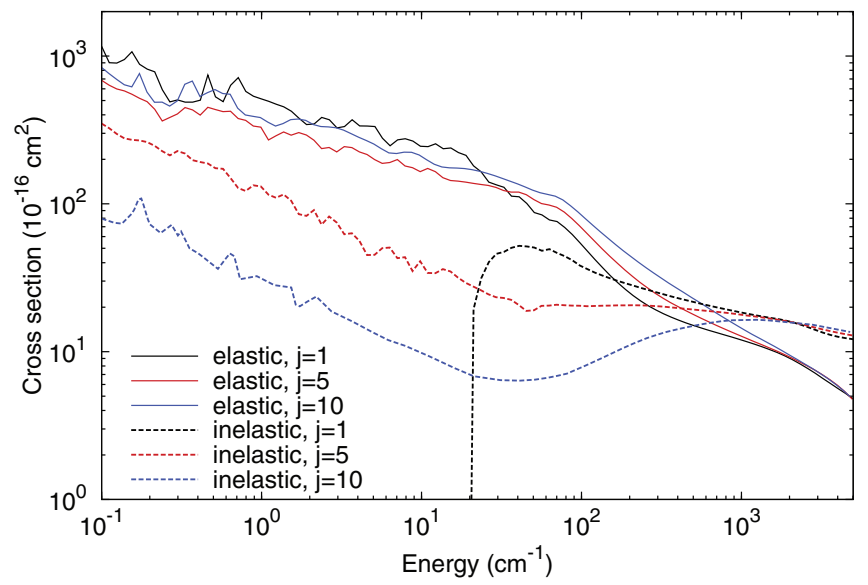

FIG. 8. Momentum transfer cross sections for the initial levels $j=1$, $j=5$, and $j=10$. Full lines, elastic cross sections; dashed lines, inelastic cross sections. 
differential cross section is large for all scattering angles. However, as the energy increases, the contributions from large $\gamma$ decrease rapidly and the collisions are dominated by forward scattering. Therefore, the momentum transfer cross sections $\sigma_{j \rightarrow j^{\prime}}^{\text {tr }}$ decrease more quickly than the integral cross sections $\sigma_{j \rightarrow j^{\prime}}$ as the energy increases. At low energy, the momentum transfer cross section is dominated by elastic scattering. However, as can be seen from Fig. 8, the contribution of inelastic scattering increases at high energy and the inelastic momentum cross section becomes larger than the elastic at energies above $10^{3} \mathrm{~cm}^{-1}$. At these energies, the cross sections for different initial rotational levels become very similar.

\section{Rate coefficients}

The scattering rate coefficients (11) are presented in Fig. 9(a) for temperatures between 1 and $1000 \mathrm{~K}$ and for the initial rotational levels $j=1, j=5$, and $j=10$. The elastic rates $k_{j j}$ are very similar for the three initial rotational levels and grow with increasing temperature. The inelastic rates (summed over all final rotational levels $j^{\prime} \neq j$ ) are displayed in the same figure. The rates are consistent with the cross sections discussed above (see Fig. 7) and increase with $T$. Over the whole range of temperatures considered, the rate coefficients are dominated by elastic collisions, as expected from the previous discussion. The momentum transfer rates for initial rotational levels $j=1,5$, and 10 are shown in Fig. 9(b). The elastic rates have comparable magnitudes, although they differ by as much as $50 \%$ at $T=1 \mathrm{~K}$. The elastic momentum transfer rate for $j=1$ increases from $1 \mathrm{~K}$ to $16 \mathrm{~K}$,

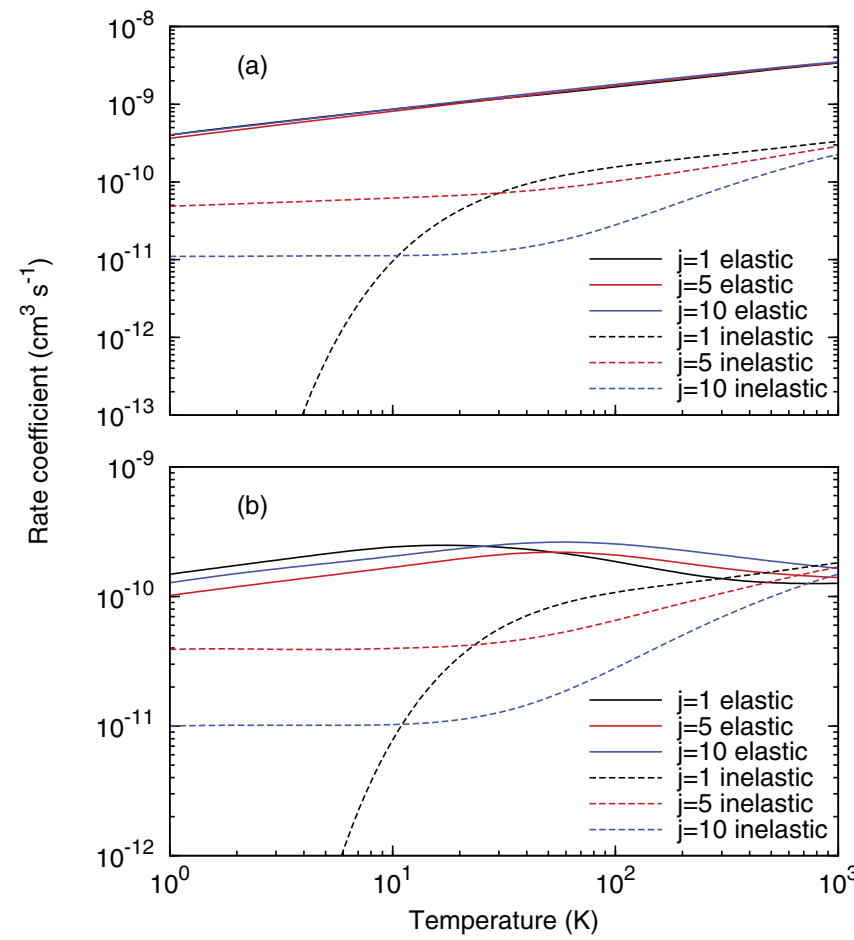

FIG. 9. Rates coefficients for $\mathrm{Ag}-\mathrm{N}_{2}$ collisions as a function of the temperature for the three initial rotational levels $j=1, j=5$, and $j=10$. (a) Scattering rate coefficients; (b) momentum transfer rate coefficients. Full lines: elastic collisions; dashed lines: inelastic collisions. where it reaches a maximum, and then decreases from $16 \mathrm{~K}$ to $1000 \mathrm{~K}$. The rate for the initial rotational levels $j=5$ and $j$ $=10$ presents a similar behavior, but the maximum is shifted toward higher temperatures. The elastic momentum transfer rates are smaller than the scattering rates, and the difference between the two types of rates increases from a factor of 3 at $T=1 \mathrm{~K}$ to a factor of 25 at $T=1000 \mathrm{~K}$. This reflects the fact that the elastic momentum transfer cross sections decrease more rapidly than the scattering cross sections when the energy increases. On the other hand, the inelastic momentum transfer rates are very similar to the inelastic scattering rates, so that inelastic transitions play a much more important role for momentum transfer rates at high temperatures. This behavior was also observed in the case of $\mathrm{Na}-\mathrm{N}_{2}$ collisions. ${ }^{3}$ At temperatures of $1000 \mathrm{~K}$ and above, the inelastic momentum transfer rates are in fact larger than the elastic rates.

\section{Comparison with $\mathrm{Na}-\mathrm{N}_{\mathbf{2}}$ scattering}

As Ag has been suggested as a proxy for $\mathrm{Na}$ in experiments with $\mathrm{N}_{2}$ and $\mathrm{O}_{2},{ }^{4}$ it is useful to compare the collisional properties of both species with $\mathrm{N}_{2}$. In Fig. 10, we show a comparison of the scattering cross sections and the rate coefficients for the initial rotational level $j=5$. We have extended the results of our previous calculations on $\mathrm{Na}-\mathrm{N}_{2}$ collisions for energies down to $0.1 \mathrm{~cm}^{-1}$ and up to $5000 \mathrm{~cm}^{-1}$ in order to be able to compare the cross sections (rates) over a large
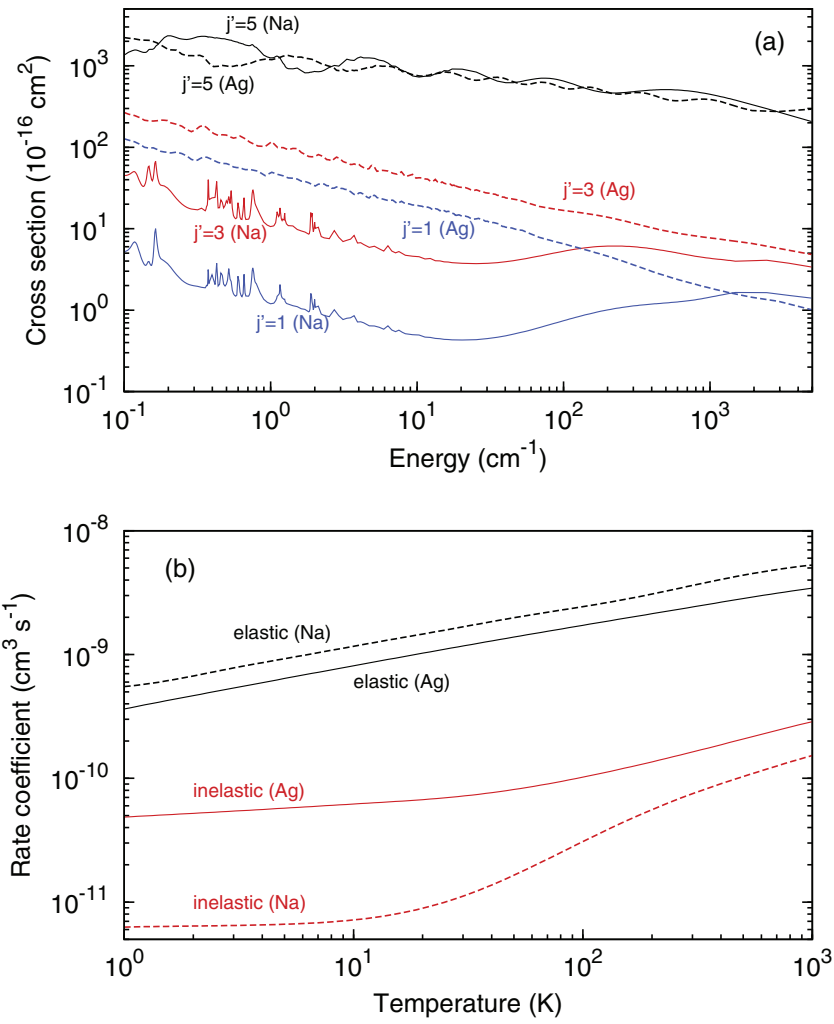

FIG. 10. Comparison between (a) the scattering cross sections, and (b) the scattering rate coefficients, in collision of $\mathrm{Ag}$ or $\mathrm{Na}$ with $\mathrm{N}_{2}(j=5)$. The value of the final rotational level $j^{\prime}$ is indicated next to each curve and the colliding atom in indicated in parentheses. Panel (a) shows the elastic cross section as well as the relaxation cross sections. Panel (b) shows the elastic and inelastic (including excitation) rate coefficients. 
range of energies (temperatures). As illustrated in Fig. 10(a), the elastic cross sections for both processes are of the same magnitude, while the inelastic cross sections are much more important in the case of $\mathrm{Ag}-\mathrm{N}_{2}$ collisions at low energy. At energies larger than $10^{3} \mathrm{~cm}^{-1}$, the relaxation cross sections become similar for the two systems. This can be explained by observing that the van der Waals potential for $\mathrm{AgN}_{2}$ is more anisotropic than in the case of $\mathrm{NaN}_{2}$, which can be seen clearly by comparing the radial functions $V_{\lambda}$ for both complexes (see Fig. 2 and Fig. 2 of Ref. 3). We observe that at low energy, the terms with $\lambda \neq 0$ are larger for the $\mathrm{AgN}_{2}$ complex than for $\mathrm{NaN}_{2}$ in the classically allowed region, therefore, favoring inelastic transitions in the former system. This discussion is also valid for the momentum transfer cross sections.

The scattering rate coefficients, shown in Fig. 10(b), reflect the above discussion on the cross sections. At the lowest temperature considered in this work $(T=1 \mathrm{~K})$, the inelastic rate for $\mathrm{Ag}-\mathrm{N}_{2}$ collisions is larger than the rate for $\mathrm{Na}-\mathrm{N}_{2}$ by an order of magnitude, while at $T=1000 \mathrm{~K}$ the difference is about a factor of 2. On the other hand, the elastic rate for $\mathrm{Na}-\mathrm{N}_{2}$ is larger than for $\mathrm{Ag}-\mathrm{N}_{2}$. This is mainly due to the fact that the reduced mass of the system, which is larger for $\mathrm{AgN}_{2}$, appears in the calculation of the rate coefficient (see Eq. (11)). The total rate (obtained by summing the elastic and inelastic rates) for the two collisional complexes is also very similar. If the mass difference between the two systems is neglected, we find that the rates for $\mathrm{Na}-\mathrm{N}_{2}$ and $\mathrm{Ag}-\mathrm{N}_{2}$ collisions differ by at most $10 \%$ over the range of temperatures considered in this work. Therefore, while the contributions of elastic and inelastic transitions are quite different, we conclude that the total rate coefficient for $\mathrm{Ag}-\mathrm{N}_{2}$ collisions can be obtained simply by scaling the rate for $\mathrm{Na}-\mathrm{N}_{2}$ collisions by the square root of the ratio of the reduced masses of the two systems. These findings are also valid in the case of the momentum transfer rate coefficients, except that the difference between the total rates is of at most $15 \%$ instead of $10 \%$.

\section{CONCLUSIONS}

We have performed a quantal study of elastic and inelastic rotational collisions of $\mathrm{Ag}$ with $\mathrm{N}_{2}$ for collision energies between 0.1 and $5000 \mathrm{~cm}^{-1}$. We have obtained the two-dimensional PES of the ground state of the $\mathrm{AgN}_{2}$ complex by means of the coupled-cluster method implemented in MOLPRO with the $\mathrm{N}_{2}$ internuclear distance fixed to its equilibrium geometry. The potential has a minimum at the geometry $R=8.13$ a.u. and $\theta=127.2^{\circ}$, while the maximum depth of the well is $81.8 \mathrm{~cm}^{-1}$. The asymptotic part of the potential was constructed analytically by calculating the $C_{6}$ dispersion coefficient.

We investigated the $\mathrm{Ag}-\mathrm{N}_{2}$ collisions using the quantummechanical close-coupling method for energies between 0.1 and $5000 \mathrm{~cm}^{-1}$. We calculated the cross sections for elastic scattering, as well as for rotational excitation and relaxation, for various initial rotational levels. Over the range of energies considered in this work, the elastic cross sections are about an order of magnitude larger than the inelastic cross sections. The elastic cross sections present a complicated resonance structure, which depends on the initial rotational level $j$ for energies below the depth of the potential well, while for energies above $80 \mathrm{~cm}^{-1}$ the elastic cross sections are very similar for all values of $j$. The inelastic cross sections, on the other hand, decrease with increasing initial rotational level but become independent of $j$ at high energy. The elastic momentum transfer cross sections are smaller than the scattering cross section, and the difference between the two types of cross sections increases with energy. However, this is not the case for the inelastic momentum transfer cross sections, so that at high energy the momentum transfer occurs primarily through inelastic collisions. Finally, we calculated the elastic and inelastic rate coefficients for temperatures between $1 \mathrm{~K}$ and $1000 \mathrm{~K}$. The elastic scattering rate increases with the temperature, while the elastic momentum transfer rate increases until it reaches a maximum at a temperature, which depends on the initial rotational level, and then decreases at higher temperatures.

\section{ACKNOWLEDGMENTS}

The authors would like to thank D. Budker for useful discussions as well as J. Mitroy and H. Sadeghpour for providing us with the dynamical electric dipole polarizabilities for the $\mathrm{Ag}$ atom. J.L. is supported by a fellowship from the Belgian American Educational Foundation, and the research of A.D. and P.Z. is supported by the Chemical Science, Geoscience, and Bioscience Division of the Office of Basic Energy Science, Office of Science, U.S. Department of Energy.

${ }^{1}$ R. Holzlöhner, S. Rochester, D. Bonaccini Calia, D. Budker, J. Higbie, and W. Hackenberg, Astron. Astrophys. 510, A20 (2010).

${ }^{2}$ J. Higbie, S. Rochester, B. Patton, R. Holzlöhner, D. Bonaccini Calia, and D. Budker, Proc. Natl. Acad. Sci. U.S.A. 108, 3522 (2011).

${ }^{3}$ J. Loreau, P. Zhang, and A. Dalgarno, J. Chem. Phys. 135, 174301 (2011).

${ }^{4}$ D. Budker, private communication (2011).

${ }^{5}$ A. Sushkov and D. Budker, Phys. Rev. A 77, 042707 (2008).

${ }^{6}$ T. Tscherbul, P. Zhang, H. R. Sadeghpour, and A. Dalgarno, Phys. Rev. Lett. 107, 023204 (2011).

${ }^{7}$ T. Walker and W. Happer, Rev. Mod. Phys. 69, 629 (1997).

${ }^{8}$ B. Lancor, E. Babcock, R. Wyllie, and T. Walker, Phys. Rev. Lett. 105, 083003 (2010).

${ }^{9}$ J. Bendtsen, J. Raman Spectrosc. 2, 133 (1974).

${ }^{10}$ P. J. Knowles, C. Hampel and H.-J. Werner, J. Chem. Phys. 99, 5219 (1993).

${ }^{11}$ J. D. Watts, J. Gauss, and R. J. Bartlett, J. Chem. Phys. 98, 8718 (1993).

${ }^{12}$ H.-J. Werner, P. J. Knowles, R. Lindh, F. R. Manby, M. Schütz et al., MOLPRO, version 2009.1, a package of ab initio programs, 2009, see http://www.molpro.net.

${ }^{13}$ K. Peterson and C. Puzzarini, Theor. Chem. Acc. 114, 283 (2005).

${ }^{14}$ D. Figgen, G. Rauhut, M. Dolg, and H. Stoll, Chem. Phys. 311, 227 (2005).

${ }^{15}$ T. H. Dunning Jr., J. Chem. Phys. 90, 1007 (1989).

${ }^{16}$ S. Boys and F. Bernardi, Mol. Phys. 19, 553 (1970).

${ }^{17}$ A. J. Stone, The Theory of Intermolecular Forces (Clarendon, 1997).

${ }^{18}$ D. M. Bishop and J. Pipin, J. Chem. Phys. 97, 3375 (1992).

${ }^{19}$ R. Moszynski, P. S. Zuchowski, and B. Jezioski, Collect. Czech. Chem. Commun. 70, 1109 (2005); T. Korona, M. Przybytek, and B. Jeziorski, Mol. Phys. 104, 2303 (2006).

${ }^{20}$ A. K. Wilson, T. V. Mourik, and T. H. Dunning Jr., J. Mol. Struct.: THEOCHEM 388, 339 (1996).

${ }^{21}$ J. Zhang, J. Mitroy, H. R. Sadeghpour, and M. Bromley, Phys. Rev. A 78, 062710 (2008)

${ }^{22}$ A. Arthurs and A. Dalgarno, Proc. R. Soc. London, Ser. A 256, 540 (1960).

${ }^{23} \mathrm{D}$. R. Flower, Molecular Collisions in the Interstellar Medium, $2 \mathrm{nd}$ ed. (Cambridge University Press, Cambridge, 2007).

${ }^{24}$ A. Lothus and P. Krupenie, J. Phys. Chem. Ref. Data 6, 113 (1977). 
${ }^{25}$ G. Parker and R. Pack, J. Chem. Phys. 68, 1585 (1978).

${ }^{26} \mathrm{~J}$. M. Hutson and S. Green, MOLSCAT computer code, version 14, distributed by Collaborative Computational Project No. 6 of the Engineering and Physical Sciences Research Council (UK), 1994.

${ }^{27}$ D. Manolopoulos and M. Alexander, J. Chem. Phys. 86, 2044 (1987).

${ }^{28}$ P. G. Burke, D. Scrutton, J. H. Tait, and A. J. Taylor, J. Phys. B 2, 1155 (1969).
${ }^{29}$ J. T. Muckerman and R. B. Bernstein, Chem. Phys. Lett. 4, 183 (1969).

${ }^{30}$ L. N. Smith, D. J. Malik, and D. Secrest, J. Chem. Phys. 71, 4502 (1979).

${ }^{31}$ R. B. Bernstein, Adv. Chem. Phys. 10, 75 (1966).

${ }^{32}$ L. D. Landau and E. M. Lifshitz, Quantum Mechanics: Non-relativistic Theory (Pergamon, 1997). 